\title{
Accurate measurement of the transition dipole moment of self-assembled quantum dots
}

Stobbe, Søren; Johansen, Jeppe; Nikolaev, I.S.; Lund-Hansen, Toke; Kristensen, Philip Trøst; Hvam, Jørn Märcher; Vos, Willem L.; Lodahl, Peter

\section{Published in:}

European Conference on Lasers and Electro-Optics, 2007 and the International Quantum Electronics Conference. CLEOE-IQEC 2007.

Link to article, DOI:

10.1109/CLEOE-IQEC.2007.4387029

Publication date:

2007

Document Version

Publisher's PDF, also known as Version of record

Link back to DTU Orbit

Citation (APA):

Stobbe, S., Johansen, J., Nikolaev, I. S., Lund-Hansen, T., Kristensen, P. T., Hvam, J. M., Vos, W. L., \& Lodahl, P. (2007). Accurate measurement of the transition dipole moment of self-assembled quantum dots. In European Conference on Lasers and Electro-Optics, 2007 and the International Quantum Electronics Conference. CLEOE-IQEC 2007. (pp. 1-1). IEEE. https://doi.org/10.1109/CLEOE-IQEC.2007.4387029

\section{General rights}

Copyright and moral rights for the publications made accessible in the public portal are retained by the authors and/or other copyright owners and it is a condition of accessing publications that users recognise and abide by the legal requirements associated with these rights.

- Users may download and print one copy of any publication from the public portal for the purpose of private study or research.

- You may not further distribute the material or use it for any profit-making activity or commercial gain

- You may freely distribute the URL identifying the publication in the public portal 


\title{
Accurate Measurement of the Transition Dipole Moment of Self-Assembled Quantum Dots
}

\author{
Soren Stobbe ${ }^{I}$, Jeppe Johansen ${ }^{I}$, Ivan S. Nikolaev ${ }^{2,3}$, Toke Lund-Hansen ${ }^{1}$, Philip T. Kristensen ${ }^{1}$, Jorn M. Hvam ${ }^{1}$, \\ Willem L. Vos ${ }^{2,3}$, Peter Lodahl ${ }^{1}$ \\ 1. COM $\bullet D T U$, Department of Communications, Optics, and Materials \\ Nano DTU, Technical University of Denmark, DTU - Building 345V, DK-2800 Kgs. Lyngby, Denmark \\ Phone: +4545256379,FAX:+4545936581,E-mail: sst@com.dtu.dk \\ 2. FOM Institute for Atomic and Molecular Physics (AMOLF), $1098 \mathrm{SJ}$ Amsterdam, The Netherlands \\ 3. Complex Photonic Systems, MESA+Research Institute, University of Twente, 7500 AE Enschede, The Netherlands
}

Self-assembled quantum dots (QDs) are highly interesting for fundamental experiments in quantum optics as well as for optical communication devices. A key parameter determining the interaction between QDs and optical fields is the optical transition dipole moment. Significant progress has been made to increase the dipole moment by engineering of the QD geometry whereby light-matter interaction is enhanced [1]. Unfortunately, the lack of an accurate technique for measuring the dipole moment has so far prevented a systematic study of the dipole moment. Here we present quantitative measurements of the dipole moment of an ensemble of self-assembled quantum dots employing a modified optical local density of states (LDOS). The LDOS is controlled by varying the distance from the QDs to a semiconductor/air interface.
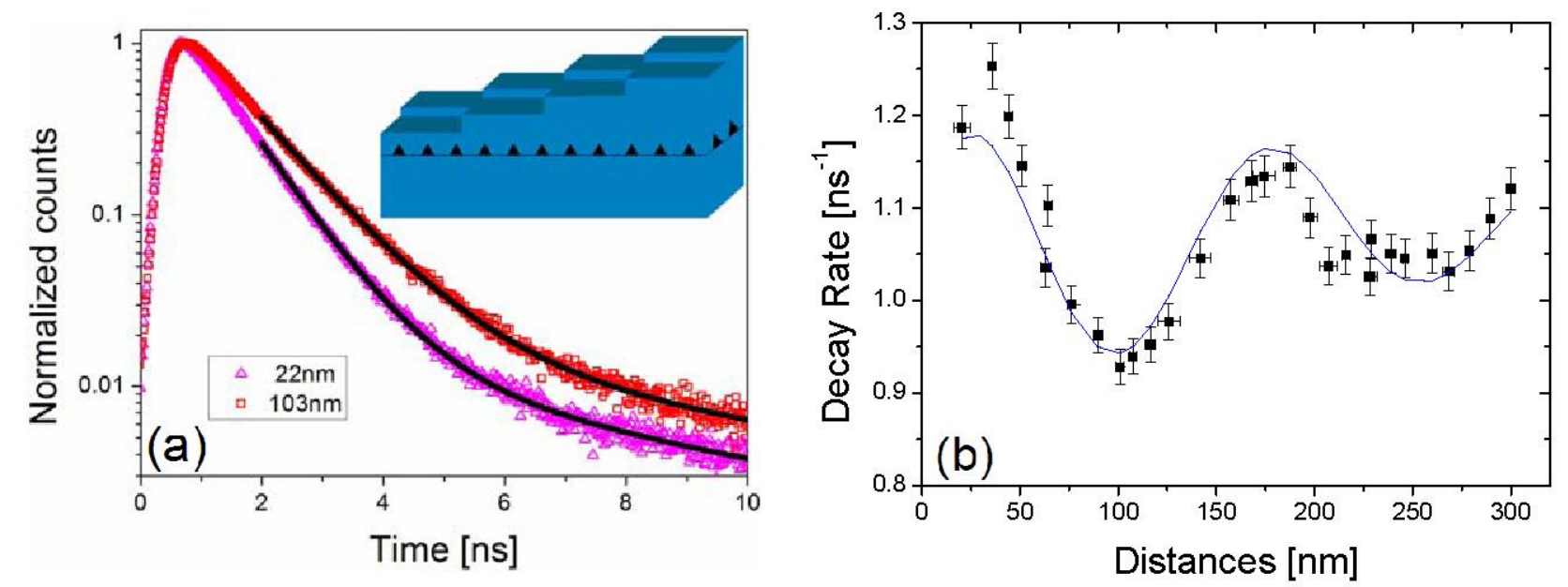

Figure 1 (a) Representative ground state decay curves for two different distances from QDs to the GaAs/air interface. The inset shows a schematic layout of the investigated sample. The surface of the wafer is etched into different heights so that the QDs (black triangles) experience a different vacuum field due to the reflection at the GaAs/air interface. (b) Measured decay rates of the fast component of the spontaneous emission from QDs for different distances to the GaAs/air interface (solid dots). The solid line is the theoretical decay rate calculated using the deduced radiative and nonradiative decay rates.

We fabricated 32 samples from a GaAs wafer containing self-assembled InAs quantum dots grown by molecular beam epitaxy. The samples were prepared using optical lithography and wet chemical etching in order to vary the distance from QDs to the GaAs/air interface, see the inset of Fig. 1(a). The reflection from the interface creates an oscillation in the LDOS which can be calculated exactly and without free parameters. From the oscillation (Fig. 1(b)) the radiative and non-radiative decay rates and the dipole moment for a homogeneous medium can be found.

The decay rate of the QD exciton ground state is obtained by time-resolved measurements of spontaneous emission. The decay curves are modelled by bi-exponential decays leading to a fast and a slow decay as seen in Fig. 1(a). The fast decay is due to the bright ground-state exciton and we find that $\Gamma_{\text {rad }}=0.84 \pm 0.08 \mathrm{~ns}^{-1}$ and $\Gamma_{\text {non-rad }}=$ $0.25 \pm 0.08 \mathrm{~ns}^{-1}$ for our InAs QDs in bulk GaAs. Using Fermi's Golden Rule the radiative decay rate in a homogeneous medium can be related to the dipole moment $d$. For these particular QDs we find $d=9.7 \pm 0.2 \times 10^{-29} \mathrm{mC}$. The slow decay is most likely due to another energy level of the ground state fine-structure giving rise to dark excitons that are dominated by non-radiative decay and therefore independent of LDOS modulation.

We stress that our method can be applied to other material systems and it therefore provides a general recipe for systematic studies of transition dipole moments of QDs. Our work will provide important input to obtain full quantitative understanding of light-matter dynamics in photonic crystals [2].

[1] J.P. Reithmaier, G. Sek, A. Löffler, C. Hofmann, S. Kuhn, S. Reitzenstein, L.V. Keldysh, V.D. Kulakovskii, T.L. Reinecke, and A. Forchel, Nature 432, 197 (2004).

[2] I.S. Nikolaev, P. Lodahl, A.F. van Driel, A.F. Koenderink, and W.L. Vos, to appear in Phys. Rev. B (2007). 